\title{
MIMO-MMSE Model using Multiple Active Antennas for Rayleigh Fading Channel
}

\author{
Rashmi Tiwari \\ Dept. of Electronics and Telecommunication \\ Jabalpur Engineering College, Jabalpur(M.P)
}

\author{
Agya Mishra, Ph. D. \\ Assistant Professor \\ Dept. of Electronics and Telecommunication \\ Jabalpur Engineering College, Jabalpur (M.P.)
}

\begin{abstract}
Spatial Modulation (SM) is a technique that can enhance the capacity of MIMO schemes by exploiting the index of transmit antenna to convey information bits. This paper describes a MIMO transmission scheme that combines SM and spatial multiplexing. In the basic form of SM, only one out of $\mathrm{N}_{\mathrm{T}}$ available antennas is selected for transmission in any given symbol interval. This paper proposes to use more than one active antenna to transmit several symbols simultaneously. This would increase the spectral efficiency and decreases BER (bit error rate) at the receiver, an MMSE detector is employed to jointly estimate the Transmitted symbols as well as the index of the active transmit antennas. The results show that the detection based on zero forcing is good for noise free channel and is successful in removing ISI, but MMSE is a better choice than ZF in terms of BER characteristics and under Noise performance. In MIMO transmission scheme the uncorrelated fading channel also called statistical model is used for the effect of a propagation environment on a radio signal. Sometimes use of multiple antenna techniques is problem depended but it used because it gives better performance like heavily built urban environment where radio signals are fade and scattered.
\end{abstract}

Keywords-Additive White Gaussian noise ,Rayleigh fading channel, Antenna Combination Binary phase shift keying (BPSK), Bit error rate (BER), Inter-symbol interference (ISI), Minimum mean square error (MMSE), MIMO System, Spatial Modulation, Spatial Multiplexing, Zero Forcing (ZF).

\section{INTRODUCTION}

In wireless communication the propagation channel is characterized by multipath propagation due to scattering on different obstacle. The multipath problem is a typical issue in communication system. In an urban environment, these signals will bounce off trees, buildings, etc. and continue on their way to their destination (the receiver) but in different directions. With MIMO, the receiving end uses an algorithm or special signal detection technique to solve the problem and multiple antennas are used to produce one signal that has the originally transmitted data. So the need of MIMO scheme is the key elements for wireless communication system.

Spatial Modulation was first proposed by Mesleh et al.. Paper [1] presents a MIMO transmission scheme based on the GSM (generalized spatial modulation) combined with the spatial multiplexing. Unlike the GSM which transmits the same symbol over the active antennas, the proposed scheme uses $\mathrm{N}_{\mathrm{A}}$ antennas out of $\mathrm{N}_{\mathrm{T}}$ to transmit $\mathrm{N}_{\mathrm{A}}$ different symbols simultaneously, where $\mathrm{N}_{\mathrm{A}}<\mathrm{N}_{\mathrm{T}}$. The scheme exploits the index of Active antennas combinations instead of antenna index to convey information bits. As results more information bits can be sent by the scheme, i.e., increased spectral efficiency. In paper [2] to deal with the constraint of the existing method, a modified detection algorithm based on Normalized maximum ratio combination (NMRC) was proposed, which can be used in unconstrained channel. Analysis and simulation results show that NMRC detection can work efficiently with very low complexity. In[3] In this paper, a new transmission approach, called spatial modulation, that entirely avoids ICI(inter channel interference) and requires no synchronization between the transmitting antennas while maintaining high spectral efficiency is presented by using a novel transmit antenna number detection algorithm called iterative-maximum ratio combining (i-MRC) is presented. The results are compared to ideal V-BLAST (Vertical-Bell Lab Layered Space-Time)[3] and to MRC. Spatial modulation outperforms MRC. The (bit-error-rate) BER performance and the achieved spectral efficiency is comparable to V-BLAST. In paper (4) this paper is mainly focused on the concept of SM to design the multiple-antenna wireless system and to get transmit-diversity gain, given by the Space-Time Block Codes (STBCs) technology.

Existing algorithms that are known from literature that is maximum likelihood detection and zero-forcing detection algorithm the receiver uses maximum likelihood (ML) detector to estimate the combination index, and transmit symbol vector. At the receiver end, a zero-forcing detection algorithm is used to estimate the transmitted symbols and the index of active antennas combination. These detection algorithms suffer from a high computational complexity Inter channel Interferences (ICI). To solve this problem, This Paper present a New MMSE detector based on the ZF detector And ML (Maximum Likelihood Detection). The result from the proposed Algorithms (MMSE) in this paper work is better as compare to the other existing algorithms which are being shown in the experimental result section- 3 of this paper. The performance improvement of this MMSE detection algorithm over the zero forcing is occurred by increasing the modulation order. Rayleigh fading channel is used to see the performance MIMO communication system. It makes a better choice for high data rate transmission for LTE-Advanced. 


\section{PROPOSED MIMO-MMSE MODEL}

In this proposed MIMO-MMSE model there is multiple antennas can be used at the transmitter and receiver end for high data rate to make a communication system better in higher scattering environments. In this MIMO model $\mathrm{N}_{T}$ number of transmitting antenna and $N_{R}$ number of receiving antennas are used where only $\mathrm{N}_{\mathrm{A}}$ number of active antennas is used to transmit the given input symbols.

In this model if only one active antenna out total transmitting antennas are used spectral efficiency of system is low. So for enhancing the spectral efficiency of communication system multiple active antennas can be used. In this paper two active antennas are used so that spectral efficiency and BER is improved compared to one active antenna.

\subsection{MIMO-Model}

In this paper consider a MIMO communications system with $\mathrm{N}_{\mathrm{T}}$ transmit antennas and $\mathrm{N}_{\mathrm{R}}$ receives antennas as shown in Fig. 1 where $b$ is a sequence of independent random bits to be transmitted over a MIMO channel. The transmitter groups the incoming bits, $b$, into blocks of $\log _{2}\left(\mathrm{Nc}^{*} \mathrm{M}^{\mathrm{NA}}\right)$ bits. The first $\log _{2}(\mathrm{Nc})$ bits are used to select the index of combination of active antennas, and the remaining $\mathrm{N}_{\mathrm{A}} \log _{2}(\mathrm{M})$ bits are mapped into a complex signal constellation vector $\mathrm{s}=\left[\mathrm{S}_{1} \mathrm{~S}_{2} \ldots \mathrm{S}_{\mathrm{NA}}\right]^{\mathrm{T}}$ to be transmitted over the $\mathrm{N}_{\mathrm{A}}$ active antennas, where $\mathrm{S}_{\mathrm{k}}(\mathrm{k}=1$, $\left.\ldots, \mathrm{N}_{\mathrm{A}}\right)$ is selected from an BPSK signal points .

At the receiver end, the received samples can be expressed as

$$
\mathrm{y}=\mathrm{H}_{\mathrm{X}}+\mathrm{n}
$$

Where $\mathrm{y}=\left[\mathrm{Y}_{1}, \mathrm{Y}_{2} \ldots Y_{N_{R}}\right]^{\mathrm{T}}$ is the $\mathrm{N}_{\mathrm{R}} \mathrm{x} 1$ received samples vector, and $\mathrm{n}=\left[\mathrm{n}_{1} \ldots n_{N_{R}}\right]^{\mathrm{T}}$ is the $\mathrm{N}_{\mathrm{R}} \times 1$ additive noise vector,
$\mathrm{H}$ is the $\mathrm{N}_{\mathrm{R}} \times \mathrm{N}_{\mathrm{T}}$ channel matrix between transmit antennas and receive antennas, and is given by:

$\mathrm{H}=\left[\begin{array}{ccc}h_{1,1} h_{1,2} & \cdots & h_{1, N_{T}} \\ \vdots & \ddots & \vdots \\ h_{N_{R}, 1} h_{N_{R, 2}} & \cdots & h_{N_{R, N} T}\end{array}\right]$

The proposed scheme converts a sequence of independent random bits into blocks. At the transmitter end each block contains $10 \mathrm{~g} 2\left(\mathrm{Nc} \mathrm{M}^{\mathrm{NA}}\right)$ bits, where $\mathrm{M}$ is the modulation order and $\mathrm{Nc}$ is the number of combinations. The first $\log _{2}\left(\mathrm{~N}_{\mathrm{C}}\right)$ bits are used to select the $\mathrm{N}_{\mathrm{T}}$ number of transmit antennas combination from the available combinations, and the next $10 \mathrm{~g} 2\left(\mathrm{M}^{\mathrm{NA}}\right)$ bits are modulated using a modulation scheme such as M -PSK and transmitted through the selected active antennas.

At the Transmitter stage in this paper consider a system with $\mathrm{N}_{\mathrm{T}}=4, \mathrm{~N}_{\mathrm{A}}=2$, and $\mathrm{M}=2$ (BPSK) [1].

In mapping procedure the system can convey Three bits in each time slots. Suppose that a block of Three bits, $\left[\begin{array}{lll}0 & 1 & 0\end{array}\right]$, is to be transmitted. In this case, the symbols -1 and 1 will be transmitted on antennas 1,2 respectively.

In Table I, illustrate a special example of the mapping of the proposed scheme for $\mathrm{N}_{\mathrm{T}}=4, \mathrm{~N}_{\mathrm{A}}=2$ and $\mathrm{M}=2$. At the receiver end this paper present a proposed algorithm that is MMSE algorithm instead of Zero-forcing algorithm because MMSE algorithm gives better Spectral efficiency and less ICI (inter-channel interference) and less error probability than Zero Forcing Algorithm

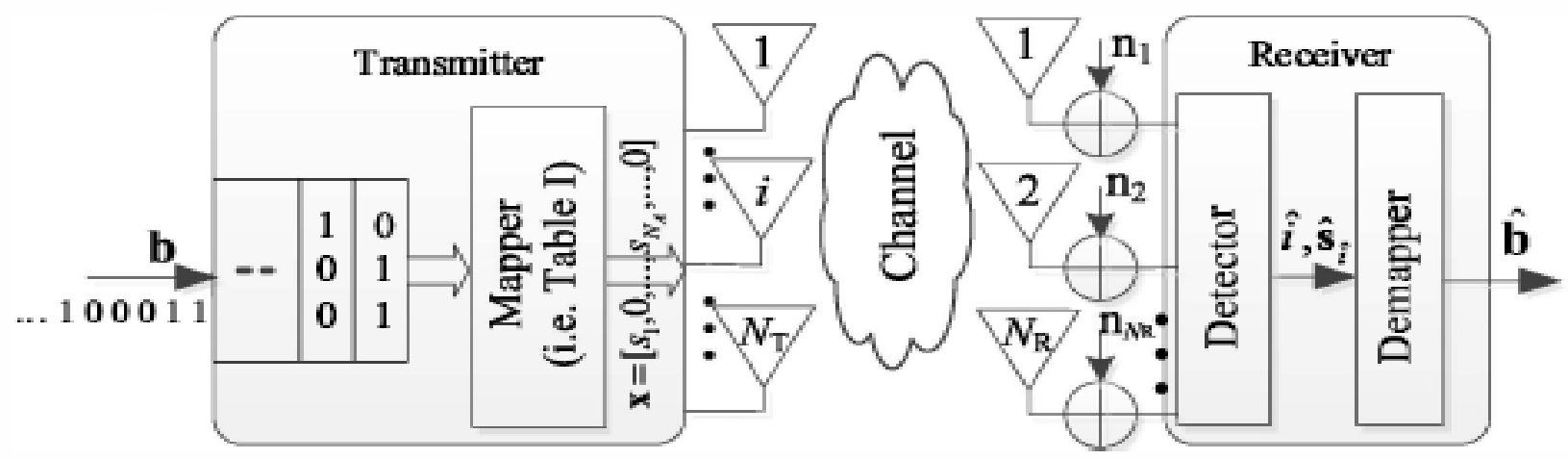

Fig.1 Block Diagram of MIMO model with MMSE Detection Algorithm [1]

Table.1 Proposed Mapping at the transmitter $\mathrm{N}_{\mathrm{T}}=4$, $\mathrm{N}_{\mathrm{R}}=4, \mathrm{~N}_{\mathrm{A}}=\mathbf{2}$

\begin{tabular}{|l|c|c|}
\hline Block Input & $\begin{array}{c}\text { Active } \\
\text { Antenna }\end{array}$ & $\begin{array}{c}\text { Transmit } \\
\text { Vector }\end{array}$ \\
\hline$\left[\begin{array}{lll}0 & 0 & 0\end{array}\right]$ & 1,2 & {$\left[\begin{array}{llll}1 & 0 & 0 & 0\end{array}\right]$} \\
\hline$\left[\begin{array}{lll}0 & 0 & 1\end{array}\right]$ & 1,2 & {$\left[\begin{array}{llll}-1 & 0 & 0 & 0\end{array}\right]$} \\
\hline$\left[\begin{array}{lll}0 & 1 & 0\end{array}\right]$ & 2,3 & {$\left[\begin{array}{llll}0 & 1 & 0 & 0\end{array}\right]$} \\
\hline$\left[\begin{array}{lll}0 & 1 & 1\end{array}\right]$ & 2,3 & {$\left[\begin{array}{llll}0 & -1 & 0 & 0\end{array}\right]$} \\
\hline$\left[\begin{array}{lll}1 & 0 & 0\end{array}\right]$ & 3,4 & {$\left[\begin{array}{llll}0 & 0 & 1 & 0\end{array}\right]$} \\
\hline$\left[\begin{array}{lll}1 & 0 & 1\end{array}\right]$ & 3,4 & {$\left[\begin{array}{lllll}0 & 0 & -1 & 0\end{array}\right]$} \\
\hline$\left[\begin{array}{lll}1 & 1 & 0\end{array}\right]$ & 4,1 & {$\left[\begin{array}{lllll}0 & 0 & 0 & 1\end{array}\right]$} \\
\hline$\left[\begin{array}{lll}1 & 1 & 1\end{array}\right]$ & 4,1 & {$\left[\begin{array}{llll}0 & 0 & 0 & -1\end{array}\right]$} \\
\hline
\end{tabular}

\subsection{Proposed Algorithm (MMSE Algorithm)}

A minimum mean square error (MMSE) estimator describes the approach which minimizes the mean square error (MSE), which is a common measure of estimator quality. The main feature of MMSE equalizer is that it does not usually eliminate ISI (inter symbol interference) completely but, minimizes the total power of the noise and ISI components in the output.

A minimum mean square error (MMSE) Algorithm describes the approach which minimizes the mean square error (MSE). The main feature of MMSE Algorithm is that it does not usually eliminate ISI completely but, minimizes the total power of the noise and ISI components in the output. Let $\mathrm{x}$ be an unknown random variable, and let y be a known random 
variable. An estimator $\mathrm{x}^{\wedge}(\mathrm{y})$ is any function of the measurement $\mathrm{y}$, and its mean square error is given by:

$\operatorname{MSE}=\mathrm{E}\left\{\left(\mathrm{x}^{\wedge}-\mathrm{x}_{2}\right)\right\}$

Where the expectation is taken over both $\mathrm{x}$ and $\mathrm{y}$.

The MMSE detector is then defined as the detector achieving minimal MSE. The linear MMSE estimator is the estimator achieving minimum MSE among all estimators of the form $\mathrm{AY}+\mathrm{b}$. If the measurement $\mathrm{Y}$ is a random vector, $\mathrm{A}$ is a matrix and $b$ is a vector.

Let us now try to understand the math for extracting the two symbols which interfered with each other. In the first time slot, the received signal on the first receive antenna is:

$$
\mathrm{y}_{1}=\mathrm{h}_{1,1} \mathrm{X}_{1}+\mathrm{h}_{1,2} \mathrm{X}_{2}+\mathrm{n}_{1}=\left(\mathrm{h}_{1,1} \mathrm{~h}_{1,2}\right)\left(\begin{array}{l}
X_{1} \\
X_{2}
\end{array}\right)
$$

The received signal on the second receive antenna is:

$$
\mathrm{y}_{2}=\mathrm{h}_{2,1} \mathrm{X}_{1}+\mathrm{h}_{2,2} \mathrm{X}_{2}+\mathrm{n}_{2}=\left(\mathrm{h}_{2,1} \mathrm{~h}_{2,2}\right)\left(\begin{array}{l}
X_{1} \\
X_{2}
\end{array}\right)
$$

Where,

$\mathrm{y}_{1}, \mathrm{y}_{2}$ are the received symbol on the first and second antenna respectively,

$\mathrm{h}_{11}$ is the channel from 1 st transmit antenna to 1 st receive antenna,

$\mathrm{h}_{12}$ is the channel from 2 nd transmit antenna to 1 st receive antenna,

$\mathrm{h}_{21}$ is the channel from 1 st transmit antenna to 2 nd receive antenna,

$\mathrm{h}_{22}$ is the channel from 2 nd transmit antenna to 2 nd receive antenna,

$\mathrm{x}_{1}, \mathrm{x}_{2}$ are the transmitted symbols and

$\mathrm{n}_{1}, \mathrm{n}_{2}$ are the noise on $1 \mathrm{st}$ and $2 \mathrm{nd}$ receive antennas. as follows:

The equation can be represented in matrix notation

$\left(\begin{array}{l}Y_{1} \\ Y_{2}\end{array}\right)=\left(\begin{array}{ll}h_{1,1} & h_{1,2} \\ h_{2,1} & h_{2,2}\end{array}\right)\left(\begin{array}{l}X_{1} \\ X_{2}\end{array}\right)+\left(\begin{array}{l}n_{1} \\ n_{2}\end{array}\right)$

Equivalently:

$$
\mathrm{y}=\mathrm{H}_{\mathrm{X}}+\mathrm{n}
$$

The Minimum Mean Square Error (MMSE) approach tries to find a coefficient $\mathrm{W}$ which minimizes the

$\mathrm{E}\left\{\left[\mathrm{w}_{\mathrm{y}-\mathrm{x}}\right]\left[\mathrm{W}_{\mathrm{y}-\mathrm{x}}\right]^{\mathrm{H}}\right\}$

Criterion,

$\mathrm{H}$ - Channel Matrix and

n - Channel noise

y- Received signal

To solve for $\mathrm{x}$, we need to find a matrix $\mathrm{W}$ which satisfies WH =I. The Minimum Mean Square Error (MMSE) detector for meeting this constraint is given by:

$\mathrm{W}=\left[\left(\mathrm{H}^{\mathrm{H}} \mathrm{H}+\mathrm{N}_{\mathrm{o}} \mathrm{I}\right)^{-1} \mathrm{H}^{\mathrm{H}}\right]$

This matrix is known as the pseudo inverse for a general $\mathrm{m} \mathrm{x}$ n matrix.

\subsection{Using Multiple Active Antennas}

Multiple-antenna techniques are a key technology for modern wireless communications, which trade-off superior error performance and higher data rates. Among the many transmission scheme that exploit multiple-antenna at the transmitter, the receiver, or both, Spatial Modulation (SM) is a recently used multiple-antenna transmission technique which can offer, with a very low system complexity, improved data rates compared to Single-Input-Single-Output (SISO) systems.

The use of multiple antennas at the transmitter and receiver MIMO system suffers from higher complexity and cost; ICI (inter channel interference) and IAS (inter Antenna synchronization).

These issues make the practical implementation of MIMO schemes difficult, especially in mobile stations, as the necessary hardware.

A new modulation concept for MIMO systems, which aims at reducing the complexity and cost of multiple-antenna schemes without reducing the end-to-end

System performance and good data rates. More specifically, the low-complexity transceiver design and high spectral efficiency are simultaneously achieved by adopting the simple modulation scheme follows' as:-

1) Just one transmit-antenna is activated for data transmission at any signaling time Instance. This is entirely avoided the ICI and IAS.

2) The spatial position of each transmit-antenna in the antenna-array is used as a source of information.

3) To increase the spectral efficiency more than one active antenna can be used at the transmitter.

\subsubsection{Combination of Active Antennas}

The proposed MMSE based spatial modulation scheme uses the index of antennas combination to transmit the information bits. In this section introduce an algorithm to design a table of active antennas combination. Consider a MIMO system with $\mathrm{N}_{T}$ and $\mathrm{N}_{R}$ transmit and receive antennas, respectively, and only $\mathrm{N}_{\mathrm{A}}$ antennas are active during transmission, where $\mathrm{N}_{\mathrm{A}} \leq$ $\mathrm{N}_{\mathrm{T}}$ Therefore, the total number of possible combinations, C, is:

$$
\mathrm{C}=\left(\begin{array}{l}
N_{T} \\
N_{A}
\end{array}\right)=\frac{N_{A} !}{N_{A} !\left(N_{T}-N_{A}\right) !}
$$

The proposed scheme uses only Nc combinations out of the total possible combinations, $\mathrm{C}$, where $\mathrm{Nc}$

$$
\mathrm{Nc}=[\mathrm{C}]_{2}{ }^{\mathrm{k}}
$$

Where $\mathrm{k}$ is an integer number. In this paper assume MIMO system with Four transmit antennas, $\mathrm{N}_{\mathrm{T}}=4$, and two antennas is active during transmission, $\mathrm{N}_{\mathrm{A}}=2$.Thus, the number of combinations that are used in the proposed scheme is $\mathrm{Nc}=6$ Therefore, the number of bits transmitted by the combination index is $\log _{2}(4)=2$ bits.

The number of bits per symbol (spectral efficiency) that can be transmitted by using the SM scheme can be calculated as:-

$$
\begin{aligned}
& \urcorner_{\mathrm{SM}}=\log _{2}\left(\mathrm{~N}_{\mathrm{C}}\right)+\log _{2}(\mathrm{M}) \\
& \text { ๆnew }=\log _{2}\left(\mathrm{~N}_{\mathrm{C}}\right)+\mathrm{N}_{\mathrm{A}} \log _{2}(\mathrm{M})
\end{aligned}
$$

If spectral efficiency is calculated by using equation(13) then it is transmitted $3 \mathrm{bps} / \mathrm{Hz}$.In this paper the new formula is used to calculate the spectral efficiency(number of bits per symbol) given in equation(14).So by increasing the active antenna spectral efficency is greatly improved i.e. 4.58 $\mathrm{bps} / \mathrm{Hz}$.

\section{EXPERIMENTAL RESULTS}

M-PSK Modulation scheme is used with $\mathrm{M}=2$ the scheme become BPSK In this section paper provides simulation results for the proposed algorithm (MMSE), and compares these results with simulation results of zero-forcing [1] .In this paper assume an uncorrelated Rayleigh fading channel, and the channel is perfectly known to the receiver. The M-PSK 
modulation if modulation order increases then MIMO-MMSE transmission model performs the best result in term of BER. To increases the spectral efficiency of this new modulation scheme in this paper two active antennas $\left(\mathrm{N}_{\mathrm{A}}=2\right)$ are used to transmit the same symbol at the same time.

\subsection{Performance of proposed model (For Spectral Efficiency Enhancement)}

In Fig. 2 plot the BER performance of the $4.58 \mathrm{bps} / \mathrm{Hz}$ spectral efficiency for the BPSK scheme with $\mathrm{N}_{\mathrm{A}}=2$, Zero Forcing and the MMSE Detector. The MIMO scheme with MMSE detector out performs the best result than Zero forcing Detector, and provides SNR gain of about $7 \mathrm{~dB}$ and $8 \mathrm{~dB}$ over the zero-forcing at BER of $0.0010,0.0004389$, respectively. The performance of this modulation scheme is degraded when the zero-forcing detector is used. Zero-forcing detection algorithm has worse performance at high SNR, since the MMSE Detector perform best at high SNR. If number of Active Antenna increases the SM Scheme gives better results.

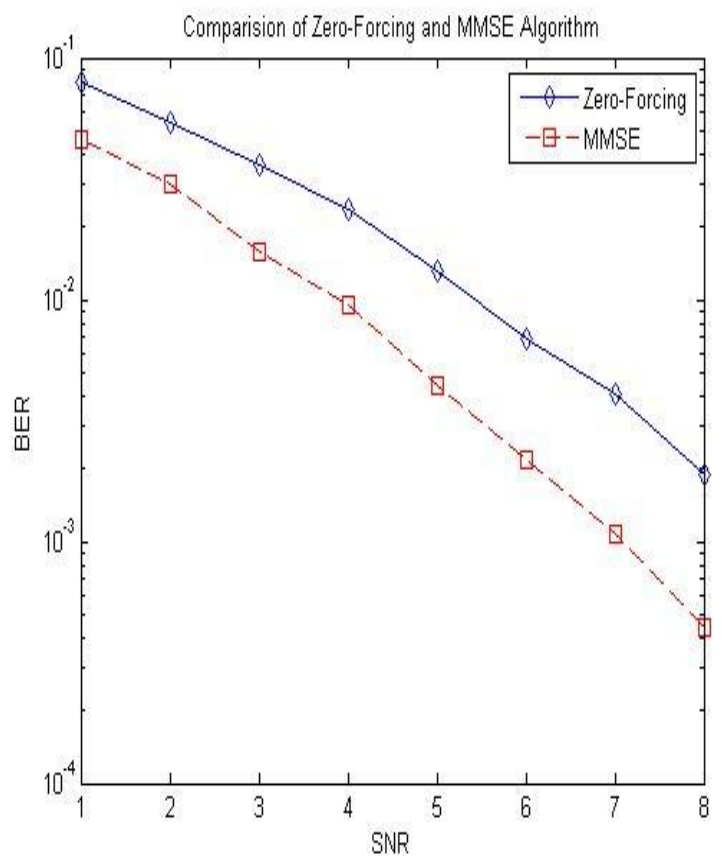

Fig.2 BER Performance for a spectral efficiency of $4.58 \mathrm{bps} / \mathrm{Hz}$

\subsection{Performance at Receiver}

In Fig.3plot the BER performance of the $4.58 \mathrm{bps} / \mathrm{Hz}$ spectral efficiency for the BPSK scheme with $\mathrm{Nr}=1, \mathrm{Nr}=2, \mathrm{Nr}=3$, $\mathrm{Nr}=4$ receiving Antennas by using the MMSE Detection Algorithm. The efficiency with MMSE detection performs the best result than Zero forcing Detector and count the minimum BER by increasing the receiving antennas.

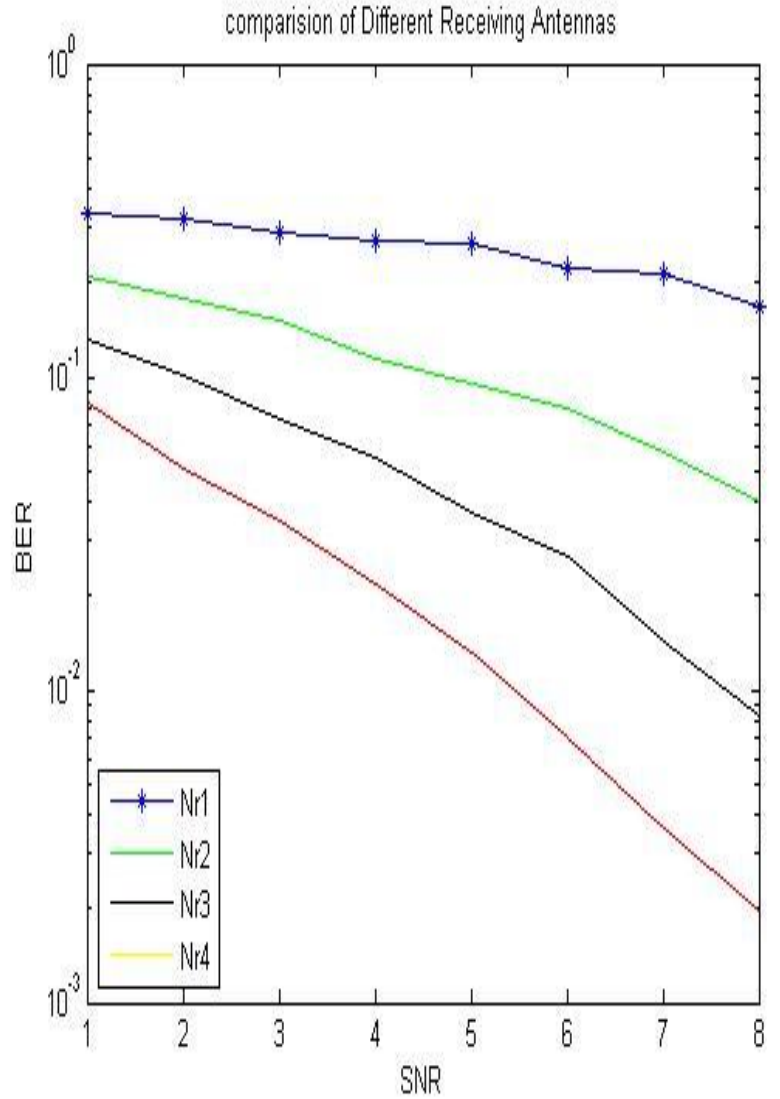

\subsection{Parameter based Analysis}

To make a better performance of MIMO systems with, different modulation schemes in terms of received energy per bit, the error-rate performance is given as function of the bit energy-to-noise density ratio $\mathrm{Eb} / \mathrm{No}$. Eb/No is the measure of signal to noise ratio for a digital communication system. It is measured at the input to the receiver and is used as the basic measure of how strong the signal is.

In fig. 4 plot the BER performance with respect to $\mathrm{Eb} / \mathrm{No}$ (energy per bit to noise power spectral density ratio). As shown in fig by increasing the parameter Eb/No the MMSE algorithm count the minimum BER and it approaches to Zero by increasing the value of $\mathrm{Eb} / \mathrm{No}$ (normalized SNR or SNR per bit). As shown in table. 2 when the value of $\mathrm{Eb} / \mathrm{No}$ is 8 the MMSE algorithm counts the minimum ber that is 0.0019 . 


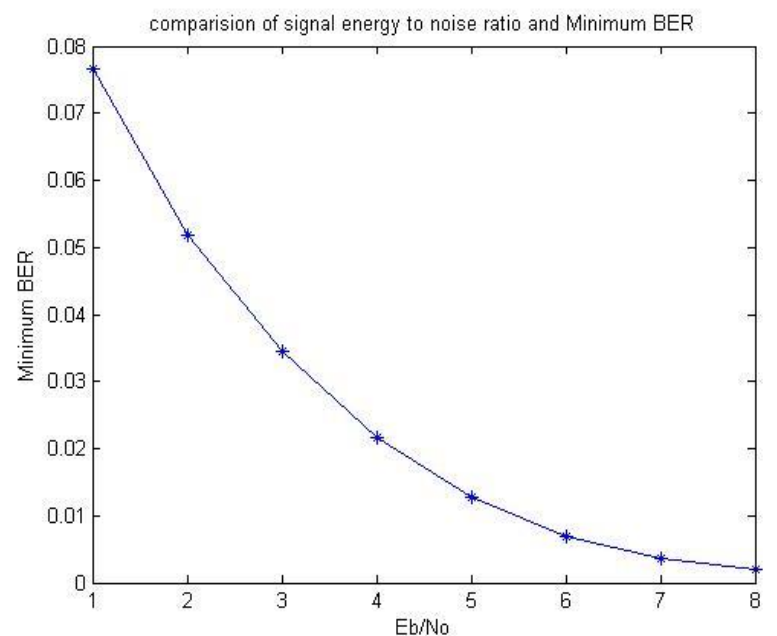

Fig.4 Performance of BER with Bit-energy to noise density ratio

Table.2 Shows Minimum BER with Eb/No

\begin{tabular}{|c|c|}
\hline Eb/No & Min BER \\
\hline 1 & 0.0767 \\
\hline 2 & 0.0519 \\
\hline 3 & 0.0345 \\
\hline 4 & 0.0217 \\
\hline 5 & 0.0128 \\
\hline 6 & 0.0070 \\
\hline 7 & 0.0036 \\
\hline 8 & 0.0019 \\
\hline
\end{tabular}

\subsection{Comparison Table between Existing and Proposed Algorithm}

\begin{tabular}{|c|c|c|c|}
\hline S.No. & Algorithms & $\begin{array}{c}\text { BER(at the } \\
\text { receiver }\end{array}$ & $\begin{array}{c}\text { BER(at the } \\
\text { transmitter) }\end{array}$ \\
\hline 1 & $\begin{array}{c}\text { Zero- } \\
\text { Forcing(sub- } \\
\text { optimal } \\
\text { )Detector }\end{array}$ & 0.0020 & 0.0037 \\
\hline 2 & $\begin{array}{c}\text { MMSE(mini } \\
\text { mum mean } \\
\text { square error) } \\
\text { Detector }\end{array}$ & 0.0019 & 0.0004389 \\
\hline
\end{tabular}

\section{CONCLUSION}

In this paper proposed MIMO-MMSE transmission scheme is presented by using multiple active antennas to improve the spectral efficiency. In the scheme combine SM with spatial multiplexing. This SM scheme uses several antennas to transmit different symbols at the same time slot, where the active antennas are subset of a larger set of transmitting antennas. By computer simulation, BER Performance for the proposed scheme is evaluated for uncorrelated Rayleigh fading channel and compared with the existing algorithm that is zero-forcing. The results show that the proposed MMSE detection algorithm performs the best result than existing. Furthermore, the performance improvement of the proposed Algorithm Over existing, increases as the transmission rate increases, which makes it is good choice for high data rate transmission systems Example WiMAX and LTE-Advanced..

\section{REFERENCES}

[1] Rajab M. Legnain, Roshdy H.M. Hafez, Ian D. Marsland "A Novel Spatial Modulation Using MIMO Spatial Multiplexing",IEEE,2013.

[2] GuoMingxi, Jia Chong, ShenYuehong,"Detection Algorithm for Spatial Modulation SystemUnder Unconstrained Channel'IEEE, 2008.

[3] R. Mesleh, and H. Haas, Chang WookAhn, and SangbohYunSpatial Modulation "A New Low Complexity Spectral Efficiency Enhancing Technique"IEEE,2006.

[4] 1Rahul Saxena, 2Prof. RupeshDubey, 3Prof. PoonamLilhare "Transmit-Diversity for Spatial Modulation (SM) and Space-Time Block CodesAReview'IJSETR, june 2013.

[5] R. Bohnke, D. Wubben, V. Kuhn, and K. Kammeyer, "Reduced complexity MMSE detection for BLAST architectures," in IEEE Global Telecommunications Conference, GLOBECOM'03., vol. 4, San Francisco, CA, USA, Dec. 2003, pp. 2258-2262

[6] J. Jeganathan, A. Ghrayeb, and L. Szczecinski, "Spatial modulation: Optimal detection and performance analysis," IEEE Communications Letters, vol. 12, no. 8, pp. 545-547, Aug. 2008.

[7] J. Jeganathan, A. Ghrayeb, and L. Szczecinski "Spatial Modulation Optimal detection and performance Analysis" IEEE communication letter vol. 12, no. 8, pp. 545-547, Aug. 2008

[8] R. Mesleh, H. Haas, C. Ahn, and S. Yun, "Spatial Modulation-a new low complexity spectral efficiency Enhancing techniques" in first international Conference on communication and networking China Com'06., Bejing, China, Oct. 2006.

[9] S. Loyka and G. Tsoulos, "Estimating MIMO System Performance using the correlation matrix aproach" IEEE communication letter vol. 6, no. 1, pp. 19-21, January 2002.

[10] R. Böhnke, D. Wübben, V. Kühn, and K. D. Kammeye "Reduced complexity MMSE detection for BLAST architectures," in Proc. IEEE Globecom'03, CA, USA, Dec. 2003 